\title{
Oxytocin receptor gene associated with the efficiency of social auditory processing
}

\section{Mattie Tops ${ }^{1,2,3}{ }^{*}$, Marinus H. van IJzendoorn ${ }^{1,2}$, Madelon M. E. Riem ${ }^{1,2}$, Maarten A. S. Boksem ${ }^{4,5}$ and Marian J. Bakermans-Kranenburg ${ }^{1,2}$}

\author{
${ }^{1}$ Centre for Child and Family Studies, Leiden University, Netherlands \\ ${ }^{2}$ Leiden Institute for Brain and Cognition, Leiden University, Leiden, Netherlands \\ ${ }^{3}$ Experimental Psychology, University of Groningen, Groningen, Netherlands \\ ${ }^{4}$ Donders Institute for Brain, Cognition and Behaviour, Radboud University, Nijmegen, Netherlands \\ ${ }^{5}$ Rotterdam School of Management, Erasmus University, Rotterdam, Netherlands
}

Edited by:

Eero Vasar, University of Tartu, Estonia

Reviewed by:

Eero Vasar, University of Tartu, Estonia

David A. Slattery, University of

Regensburg, Germany

*Correspondence:

Mattie Tops, Centre for Child and

Family Studies, University of Leiden,

Post box 9555, 2300 RB Leiden,

Netherlands.

e-mail: topsm@fsw.leidenuniv.nl
Oxytocin has been shown to facilitate social aspects of sensory processing, thereby enhancing social communicative behaviors and empathy. Here we report that compared to the AA/AG genotypes, the presumably more efficient $G G$ genotype of an oxytocin receptor gene polymorphism (OXTR rs53576) that has previously been associated with increased sensitivity of social processing is related to less self-reported difficulty in hearing and understanding people when there is background noise. The present result extends associations between oxytocin and social processing to the auditory and vocal domain. We discuss the relevance of our findings for autistic spectrum disorders (ASD), as ASD seems related to specific impairments in the orienting to, and selection of speech sounds from background noise, and some social processing impairments in patients with ASD have been found responsive to oxytocin treatment.

Keywords: oxytocin, OXTR, auditory processing, autism

\section{INTRODUCTION}

Recently there has been an upsurge in studies on the involvement of the neuropeptide oxytocin in socio-emotive processing in humans. Intranasally administered oxytocin has been shown to stimulate a range of social behaviors, including empathy (Domes et al., 2007), trust (Kosfeld et al., 2005), and in-group altruism (De Dreu et al., 2010). On the genetic level individuals with the presumably more efficient variant (GG) of an oxytocin receptor gene polymorphism (OXTR rs53576) show reduced levels of stress and increased levels of empathy. Several studies report associations of the OXTR rs53576 with stress-related and psychological traits, with the majority suggesting that carriers of the A allele (i.e., AG and AA genotypes) have an increased sensitivity to stress, reduced social skills, and more negative mental health outcomes relative to individuals with two copies of the $G$ allele (Bakermans-Kranenburg and Van IJzendoorn, 2008; Lucht et al., 2009; Rodrigues et al., 2009; Kim et al., 2010, 2011; Tost et al., 2010; Riem et al., 2011a; Saphire-Bernstein et al., 2011; but see Costa et al., 2009). For instance, Bakermans-Kranenburg and Van IJzendoorn (2008) found that mothers with the GG genotype were more sensitive to their toddlers' signals than mothers with the AA or AG genotype. In another study, we found that healthy adults with the GG genotype had more pronounced physiological reactivity (heart rate) to infant crying (Riem et al., 2011a).

Oxytocin has been shown to facilitate social aspects of sensory processing, thereby facilitating social communicative behaviors. Such effects have been demonstrated both in healthy humans and in populations that show deficiencies in social sensory processing and communication, such as individuals with autistic spectrum disorders (ASD). We recently found that intranasal application of oxytocin to healthy female volunteers increased brain responses to infant crying sounds in the inferior frontal gyrus (IFG) - anterior insula area while decreasing responses in the amygdala (Riem et al., 2011b). Other studies have found that oxytocin administration enhances autistic adults' ability to comprehend emotions in speech (Hollander et al., 2007). Oxytocin also helps autistic children better discern people's intentions by reading their eyes (Guastella et al., 2010). A specific impairment in autism appears to be orienting to, and selection of speech sounds from background speech or non-speech sounds, an impairment that is generally not accompanied by basic sensory-level or physiological hearing impairments (Ceponiene et al., 2003; Alcántara et al., 2004; Lepistö et al., 2005). Until now, effects of oxytocin on this specific aspect of auditory processing that is very important for human vocal communication have not been studied.

Here we investigated whether there is an association between the OXTR GG (relative to AG/AA) genotype and reporting less difficulty in hearing and understanding people when there is background noise. We also collected scores on measures of social desirability, neuroticism, and propensity to be discomforted by intense sensory stimulation to control for more general propensity to report complaints.

\section{MATERIALS AND METHODS PARTICIPANTS}

As part of a functional magnetic resonance imaging study reported elsewhere (Riem et al., 2011b), participants were selected from a larger study investigating caregiving responses and physiological 
reactivity to infant crying (Out et al., 2010). The original sample consisted of 50 male and 134 female adult twin pairs. Twin pairs were asked to take part in the current study if they were females without children of their own, in good health, without hearing impairments or MRI contraindications, pregnancy, and without psychiatric or neurological disorders. They were screened for alcohol and drug use. A group of 45 right-handed females were recruited, 22 from monozygotic (MZ) twin pairs, and 23 from dizygotic (DZ) twin pairs. Twin siblings of 11 participants did not participate due to MRI contraindications or other exclusion criteria. Because of this, the final sample of 45 participants consisted of 34 participants from twin pairs (10 MZ, $7 \mathrm{DZ})$ and 11 participants without twin sibling. The mean age of the participants was 29.0 years ( $S D=7.4$, range 22-49). The majority of the participants were born in the Netherlands and from Western European descent (93.8\%). Their mean educational level was 3.63 $(\mathrm{SD}=0.85)$ on a scale ranging from one (elementary school) to seven (Bachelor's or Master's degree). Permission for this study was obtained from the Leiden University Medical Center ethics committee and all participants filled out the approved informed consent form.

\section{PROCEDURE AND MEASURES}

Within 2 weeks after taking part in the fMRI study, the participants completed the self-report measures at home and returned them by mail. The questionnaire on level of difficulty hearing and understanding people consisted of four items (the item showing the largest effect: "When there is background noise, it is harder for me than for most people to understand others"; further items: "It is harder for me than for most people to understand others," "I have difficulty hearing high-pitched sounds," "I have difficulty hearing low-pitched sounds") that were rated on a 7-point rating scale ( $1=$ "I totally disagree," 7 = "I totally agree"). Cronbach's alpha was 0.84 . Scores were summed to yield a total score. Three measures were included to control for a more general propensity to report complaints. The Lie scale from the eysenck personality questionnaire (EPQ-RSS) was administered to obtain social desirability scores (Sanderman et al., 1995). The Lie scale examines social desirability and people's tendency to "fake good." This scale consists of 12 questions (Cronbach's alpha was 0.78 ). Negative affect related to intensity, rate or complexity of sensory stimulation (e.g., "Loud music makes me uncomfortable") and was measured using the six-item Discomfort scale of the Adult Temperament Questionnaire (Hartman and Rothbart, 2001). Cronbach's alpha was 0.79 . Social desirability and Negative affect were rated on scales ranging from one ("I totally disagree") to seven ("I totally agree"). We used the five factor personality inventory (FFPI) to assess $\mathrm{Neu}$ roticism [15]. The 20 items are answered on a scale from one ("not at all applicable") to five ("very much applicable"). Total scores relative to an average population score of zero were computed using the scoring software available from the FFPI authors (Hendriks et al., 1999). Raw-score Cronbach's alpha was 0.83 .

\section{GENOTYPING}

Buccal swabs from the participants were collected in lysis buffer (100 mM NaCl, $10 \mathrm{mM}$ EDTA, $10 \mathrm{mM}$ Tris $\mathrm{pH} 8,0.1 \mathrm{mg} \mathrm{ml}^{-1}$ proteinase $\mathrm{K}$ and $0.5 \% \mathrm{w} / \mathrm{v}$ SDS) until further processing. Genomic
DNA was isolated from the samples using the Chemagic buccal swab kit on a Chemagen Module I workstation (Chemagen Biopolymer Technologie AG, Baesweiler, Germany). DNA concentrations were measured using the Quant-iT DNA Assay kit (Invitrogen, Breda, the Netherlands). The average yield was $4 \mu \mathrm{g}$ of genomic DNA per buccal swab sample. The region of interest from the OXTR gene (OXTR rs53576) was amplified by polymerase chain reaction (PCR) using a forward primer (5'-GCCCACCATGCTCTCCACATC- $\left.3^{\prime}\right)$ and a reverse primer (5'-GCTGGACTCAGGAGGAATAGGGAC-3'). Typical PCR reactions contained between 10 and 100 ng genomic DNA template, $10 \mathrm{pmol}$ of forward and reverse primers. PCR was carried out in the presence of $5 \%$ dimethyl sulfoxide with $0.3 \mathrm{U}$ of BioTherm $\mathrm{AB}$ polymerase (GeneCraft, Munster, Germany) in a total volume of $30 \mu \mathrm{l}$ using the following cycling conditions: initial denaturation step of $3 \mathrm{~min}$ at $95^{\circ} \mathrm{C}$, followed by 40 cycles of $30 \mathrm{~s}$ at $95^{\circ} \mathrm{C}, 30 \mathrm{~s}$ at $60^{\circ} \mathrm{C}, 1 \mathrm{~min}$ at $72^{\circ} \mathrm{C}$ and a final extension step of $3 \mathrm{~min}$ at $72^{\circ} \mathrm{C}$. To determine the $\mathrm{A} / \mathrm{G}$ polymorphism, $\mathrm{PCR}$ fragments were sequenced using the forward primer and dye terminator chemistry (BigDye v3.1, Applied Biosystems). The genotype distribution ( $n=4 \mathrm{AA}$, $n=22 \mathrm{AG}, n=19 \mathrm{GG}$ ) was in Hardy-Weinberg equilibrium, $\chi^{2}$ $(2, N=43)=0.45, n . s$. Because of the skewed distribution, AA and $A G$ genotypes were combined in the analyses.

\section{RESULTS}

Social desirability $(M=54, \mathrm{SD}=11)$, Neuroticism $(M=-3.9$, $\mathrm{SD}=0.5)$, and Discomfort $(M=22, \mathrm{SD}=6)$ were within normal ranges for healthy populations and were not significantly correlated with difficulty hearing scores and did not differ between genotypes (all ps $>0.10$ ) and inclusion into the analyses did not produce noticeable differences in results. Participants with the GG genotype were younger $[t(42.7)=2.28, p=0.028]$, and older participants reported more difficulties hearing and understanding $(r=0.34, p=0.023)$. Because of most twin pairs both sisters took part we used the Huber-White estimates to correct for clustering of the data. We included the estimates in the Complex Sample General Linear Model (CSGLM, SPSS 17) with hearing difficulty scores as dependent variable and genotype (GG vs. AA/AG) as predictor. Participants with the GG genotype reported less difficulty hearing and understanding (difficulty hearing total score: $M=9.2$, $\mathrm{SD}=4.2)$ than participants with $\mathrm{AA} / \mathrm{AG}$ genotypes $[M=13.9$, $\left.\mathrm{SD}=3.8 ; F(1,27)=14.03, p<0.001, R^{2}=0.26\right]$. Controlling for age by including it as a second (non-significant, $p=0.160$ ) predictor in the analysis did only slightly attenuate the relationship between genotype and difficulty hearing scores, $F(1,27)=10.51$, $p=0.003$.

\section{DISCUSSION}

Our finding that individuals with the presumably more efficient GG genotype of the oxytocin receptor gene (OXTR rs53576) polymorphism show lower levels of difficulty hearing and understanding people in noisy environments than participants with the AA or AG genotypes suggests that previously reported associations of oxytocin with social processing and communication in humans can be extended to the vocal domain. Participants with different genotypes did not seem to differ in their general propensity to report complaints. Many social species vocalize, but facilitation of 
vocal communication by oxytocin had not been studied in humans before (Seltzer et al., 2010). Our results suggest that oxytocinergic mechanisms may modulate vocal processing, but provide no information about which levels of processing may be affected. However, the present result is one in a line of findings suggesting that the processing aspects that are facilitated by oxytocin are often impaired in ASD. Based on these results, discussing which levels of processing are affected in ASD may provide important information for future studies aiming at delineating more precisely possible associations between oxytocin and vocal communication.

Autistic spectrum disorders comprise a continuum of psychosocial disorders clinically characterized by social difficulties, impaired communication skills, and repetitive behavioral patterns. Despite the prevalence of ASD, the neurobiology of this disorder is poorly understood. There is ample evidence that auditory dysfunction is a common feature of ASD. In ASD malformation of nuclei within the human superior olivary complex has been reported (Kulesza et al., 2011). A study of oxytocinergic neurons in the mustached bat showed numerous terminations in this important auditory brainstem center, suggesting a neuromodulatory function of oxytocin in sensory processing (Kanwal and Rao, 2002). However, most ASD patients do not show hearing impairments at lower physiological levels, but rather seem specifically impaired in the orienting to, and selection of speech sounds from background speech or non-speech sounds (Ceponiene et al., 2003; Alcántara et al., 2004; Lepistö et al., 2005).

Recent studies showed that a control area of the ventral orienting attentional system, the IFG, is increasingly activated and functionally connected to sensory saliency areas when further evaluative processing of social or communicational auditory stimuli is necessary because of stimulus degradation, ambiguity, or noise (Frye et al., 2010; Leitman et al., 2010). We recently found that intranasal application of oxytocin or placebo to the present sample of healthy female volunteers, matched on OXTR genotypes between treatment conditions, increased brain responses to infant crying sounds in the IFG - anterior insula area while decreasing responses in the amygdala, suggesting that oxytocin increased elaborate evaluative processing of the crying sounds embedded in the scanner noise (Riem et al., 2011b). Decreased oxytocinergic facilitation of this elaborative processing or of selection of social communicative stimuli in noise, and perhaps of autonomic feedback in response to such stimuli (Riem et al., 2011a), could potentially explain selective social communicative deficits in ASD. Moreover, as the IFG is part of the hypothesized mirror neuron system and has been implicated in emotional empathy (Jabbi et al., 2007) the present result suggests that decreased oxytocin function may be associated with decreased empathy partly

\section{REFERENCES}

Alcántara, J. I., Weisblatt, E. J., Moore, B. C., and Bolton, P. F. (2004). Speech-in-noise perception in high-functioning individuals with autism or Asperger's syndrome. J. Child Psychol. Psychiatry 45, 1107-1114.

Bakermans-Kranenburg, M. J., and Van IJzendoorn, M. H. (2008). Oxytocin receptor OXTR and serotonin transporter 5-HTT genes associated with observed parenting. Soc. Cogn. Affect. Neurosci. 3, 128-134.

Baron-Cohen, S. (2002). The extreme male brain theory of autism. Trends Cogn. Sci. 6, 248-254.

Ceponiene, R., Lepistö, T., Shestakova, A., Vanhala, R., Alku, P., Näätänen, R., and Yaguchi, K. (2003). Speech-sound-selective

through a mechanism that operates already at a very basic level of the mechanisms underlying empathy: Decreased social emotional input results in less emotional information to be mirrored or empathized with (Tops, 2010).

The present study converges with patient, non-patient, and preclinical studies on the suggestion that oxytocinergic systems may be involved in modulating aspects of social processing. If this fact results from slight different in auditory capacity, language skills, or auditory processing is not know. In fact, in the present study using self-reported difficulty hearing, we cannot rule out the possibility that people without the GG allele simply believe they have more difficulty hearing. However, the hypothesis is pertinent and in agreement with previous studies. Given the fact that the great majority of studies on oxytocin have focused attention on facial and emotional expression processing, the present study add interesting data and could stimulate research on auditory and human voice processing and oxytocin.

While introducing a fascinating and plausible hypothesis regarding the role of OXTR genotype in basic auditory processing with an eye toward understanding the symptoms of ASD, the present results should be regarded preliminary. Although similar studies include more participants, the present selection of participants was homogenous on many relevant variables such as gender, ethnicity, nulliparity, and mental and physical health (Riem et al., 2011b). A homogenous sample allows for detecting smaller associations than in larger but heterogeneous samples because the influence of unmeasured third variables may be less. However, this is at the expense of the generalizability of the results to other samples. For instance, the strong impact of oxytocin on female social behavior (Young and Wang, 2004), and the disproportionately high risk of men for social disorders (Baron-Cohen, 2002), suggest sex dependent genotype effects (Tost et al., 2010). Thus it needs to be determined whether the present findings in females can be replicated in a male sample. If the conclusions are borne out in other samples and with robust measures of social auditory processing, then this could represent a major finding regarding the role of the OXTR gene in regulating human behavior.

\section{ACKNOWLEDGMENTS}

We thank the twins who participated in the study. Marian J. Bakermans-Kranenburg, Mattie Tops, and Marinus H. van IJzendoorn were supported by awards from the Netherlands Organization for Scientific Research (NWO; www.nwo.nl; Mattie Tops: VENI grant; Marian J. Bakermans-Kranenburg: VIDI and VICI grants; Marinus H. van IJzendoorn: SPINOZA prize). The funders had no role in study design, data collection and analysis, decision to publish, or preparation of the manuscript.

auditory impairment in children with autism: they can perceive but do not attend. Proc. Natl. Acad. Sci. U.S.A. 100, 5567-5572.

Costa, B., Pini, S., Gabelloni, P., Abelli, M., Lari, L., Cardini, A., Muti, M., Gesi, C., Landi, S., Galderisi, S., Mucci, A., Lucacchini, A., Cassano, G. B., and Martini, C. (2009). Oxytocin receptor polymorphisms and adult attachment style in patients with depression. Psychoneuroendocrinology 34, 1506-1514.

De Dreu, C. K. W., Greer, L. L., Handgraaf, M. J. J., Shalvi, S., Van Kleef, G. A., Baas, M., Ten Velden, F. S., Van Dijk, E., and Feith, S. W. W. (2010). The neuropeptide oxytocin regulates parochial altruism in intergroup conflict among humans. Science 328, 1408-1411. 
Domes, G., Heinrichs, M., Michel, A., Berger, C., and Herpertz, S. C. (2007). Oxytocin improves "mindreading" in humans. Biol. Psychiatry 61, 731-733.

Frye, R. E., Wu, M., Liederman, J., and McGraw Fisher, J. (2010). Greater pre-stimulus effective connectivity from the left inferior frontal area to other areas is associated with better phonological decoding in dyslexic readers. Front. Syst. Neurosci. 4:156. doi:10.3389/fnsys.2010. 00156

Guastella, A. J., Einfeld, S. L, Gray, K. M., Rinehart, N. J., Tonge, B. J., Lambert, T. J., and Hickie, I. B. (2010). Intranasal oxytocin improves emotion recognition for youth with autism spectrum disorders. Biol. Psychiatry 67, 692-694.

Hartman, C. A., and Rothbart, M. K. (2001). Dutch Translation of the Adult Temperament Questionnaire. Groningen: Department of Psychiatry, Internal publication ARIADNE research group.

Hendriks, J., Hofstee, W. K. B., and De Raad, B. (1999). The five-factor personality inventory (FFPI). Pers. Individ. Dif. 27, 307-325.

Hollander, E., Bartz, J., Chaplin, W., Phillips, A., Sumner, J., Soorya, L., Anagnostou, E., and Wasserman, S. (2007). Oxytocin increases retention of social cognition in autism. Biol. Psychiatry 61, 498-503.

Jabbi, M., Swart, M., and Keysers, C. (2007). Empathy for positive and negative emotions in the gustatory cortex. Neuroimage 34, 1744-1753.

Kanwal, J. S., and Rao, P. D. (2002). Oxytocin within auditory nuclei: a neuromodulatory function in sensory processing? Neuroreport 13, 2193-2197.

Kim, H. S., Sherman, D. K., Mojaverian, T., Sasaki, J. Y., Park, J., Suh,
E. M., and Taylor, S. E. (2011). Gene-culture interaction: Oxytocin receptor polymorphism (OXTR) and emotion regulation. Soc. Psychol. Personal. Sci. doi:10.1177/1948550611405854

Kim, H. S., Sherman, D. K., Sasaki, J. Y., Xu, J., Chu, T. Q., Ryu, C., Suh, E. M., Graham, K., and Taylor, S. E. (2010). Culture, distress, and oxytocin receptor polymorphism (OXTR) interact to influence emotional support seeking. Proc. Natl. Acad. Sci. U.S.A. 107, 15717-15721.

Kosfeld, M., Heinrichs, M., Zak, P. J., Fischbacher, U., and Fehr, E. (2005). Oxytocin increases trust in humans. Nature 435, 673-676.

Kulesza, R. J. Jr., Lukose, R., and Stevens, L. V. (2011). Malformation of the human superior olive in autistic spectrum disorders. Brain Res. 1367, 360-371.

Leitman, D. I., Wolf, D. H., Ragland, J. D., Laukka, P., Loughead, J., Valdez, J. N., Javitt, D. C., Turetsky, B. I., and Gur, R. C. (2010). "It's not what you say, but how you say it": a reciprocal temporofrontal network for affective prosody. Front. Hum. Neurosci. 4:19. doi:10.3389/fnhum.2010.00019

Lepistö, T., Kujala, T., Vanhala, R., Alku, P., Huotilainen, M., and Näätänen, R. (2005). The discrimination of and orienting to speech and non-speech sounds in children with autism. Brain Res. 1066, 147-157.

Lucht, M. J., Barnow, S., Sonnenfeld, C., Rosenberger, A., Grabe, H. J., Schroeder, W., Völzke, H., Freyberger, H. J., Herrmann, F. H., Kroemer, H., and Rosskopf, D. (2009). Associations between the oxytocin receptor gene (OXTR) and affect, loneliness and intelligence in normal subjects. Prog. Neuropsychopharmacol. Biol. Psychiatry 33, 860-866.
Out, D., Pieper, S., BakermansKranenburg, M. J., Zeskind, P. S., and van Ijzendoorn, M. H. (2010). Intended sensitive and harsh caregiving responses to infant crying: the role of cry pitch and perceived urgency in an adult twin sample. Child Abuse Negl. 34 863-873.

Riem, M. M., Pieper, S., Out, D. Bakermans-Kranenburg, M. J., and van Ijzendoorn, M. H. (2011a). Oxytocin receptor gene and depressive symptoms associated with physiological reactivity to infant crying. Soc. Cogn. Affect. Neurosci. 6, 294-300.

Riem, M. M. E., BakermansKranenburg, M. J., Pieper, S., Tops, M., Boksem, M. A. S., Vermeiren, R. R. J. M., van IJzendoorn M. H., and Rombouts, S. A. R. B. (2011b). Oxytocin modulates amygdala, insula and inferior frontal gyrus responses to infant crying: a randomized control trial. Biol. Psychiatry 70, 291-297.

Rodrigues, S. M., Saslow, L. R., Garcia, N., John, O. P., and Keltner, D. (2009). Oxytocin receptor genetic variation relates to empathy and stress reactivity in humans. Proc. Natl. Acad. Sci. U.S.A. 106 21437-21441

Sanderman, R., Arrindell, W. A., Ranchor, A. V., Eysenck, H. J., and Eysenck, S. B. G. (1995). Manual to the Dutch Eysenck Personality Questionnaire. Groningen: Health Center, University of Groningen.

Saphire-Bernstein, S., Way, B. M., Kim, H. S., Sherman, D. K., and Taylor, S. E. (2011). Oxytocin receptor gene (OXTR) is related to psychological resources. Proc. Natl. Acad. Sci. U.S.A. 108, 15118-15122.

Seltzer, L. J., Ziegler, T. E., and Pollak, S. D. (2010). Social vocalizations can release oxytocin in humans. Proc. Biol. Sci. 277, 2661-2666.

Tops, M. (2010). Oxytocin: envy or engagement in others? Biol. Psychiatry 67, e5-e6.

Tost, H., Kolachana, B., Hakimi, S., Lemaitre, H., Verchinski, B. A. Mattay, V. S., Weinberger, D. R., and Meyer-Lindenberg, A. (2010). A common allele in the oxytocin receptor gene (OXTR) impacts prosocial temperament and human hypothalamic-limbic structure and function. Proc. Natl. Acad. Sci. U.S.A. 107, 13936-13941.

Young, L. J., and Wang, Z. (2004). The neurobiology of pair bonding. Nat. Neurosci. 7, 1048-1054.

Conflict of Interest Statement: The authors declare that the research was conducted in the absence of any commercial or financial relationships that could be construed as a potential conflict of interest.

Received: 19 August 2011; accepted: 18 October 2011; published online: 04 November 2011.

Citation: Tops $M$, van IJzendoorn MH, Riem MME, Boksem MAS and Bakermans-Kranenburg MJ (2011) Oxytocin receptor gene associated with the efficiency of social auditory processing. Front. Psychiatry 2:60. doi: 10.3389/fpsyt.2011.00060

This article was submitted to Frontiers in Psychopharmacology, a specialty of Frontiers in Psychiatry.

Copyright (c) 2011 Tops, van IJzendoorn, Riem, Boksem and BakermansKranenburg. This is an open-access article subject to a non-exclusive license between the authors and Frontiers Media $S A$, which permits use, distribution and reproduction in other forums, provided the original authors and source are credited and other Frontiers conditions are complied with. 